Disclosure of Interests: Maria-Antonietta D’Agostino Speakers bureau: Sanofi, Novartis, BMS, Janssen, Celgene, AbbVie, UCB pharma and Eli Lilly, Consultant of: Sanofi, Novartis, BMS, Janssen, Celgene, AbbVie, UCB pharma and Eli Lilly, Philip G Conaghan Speakers bureau: AbbVie, AstraZeneca, BMS, Eli Lilly, Galapagos, Gilead, Novartis and Pfizer, Consultant of: AbbVie, AstraZeneca, BMS, Eli Lilly, Galapagos, Gilead, Novartis and Pfizer, Corine Gaillez Shareholder of: Novartis and BMS, Employee of: Novartis, Maarten Boers Consultant of: BMS, Novartis, Pfizer, and GSK, Esperanza Naredo Speakers bureau: AbbVie, Roche, BMS, Pfizer, UCB, Eli Lilly, Novartis, Janssen and Celgene, Consultant of: AbbVie, Novartis and BMS, Grant/research support from: Eli Lilly, Philippe Carron Speakers bureau: Pfizer, MSD, Novartis, BMS, AbbVie, UCB, Eli Lilly, Gilead and Celgene, Consultant of: Pfizer, MSD, Novartis, BMS, AbbVie, UCB, Eli Lilly, Gilead and Celgene, Grant/research support from: UCB, MSD and Pfizer, Petra Hanova: None declared, Tomás Cazenave: None declared, Catherine Bakewell Speakers bureau: AbbVie, Novartis, Sanofi Genzyme, and consulting honoraria from Pfizer, UCB, and Janssen, Consultant of: AbbVie, Novartis, Sanofi Genzyme, and consulting honoraria from Pfizer, UCB, and Janssen, Anne-Marie Duggan Employee of: Novartis, Punit Goyanka Employee of: Novartis, Georg Schett Speakers bureau: AbbVie, BMS, Celgene, Gilead, Janssen, Eli Lilly, Novartis, Roche and UCB pharma

Figure: Global OMERACT-US enthesitis score change from baseline to Week 12 and from Week 12 to 24
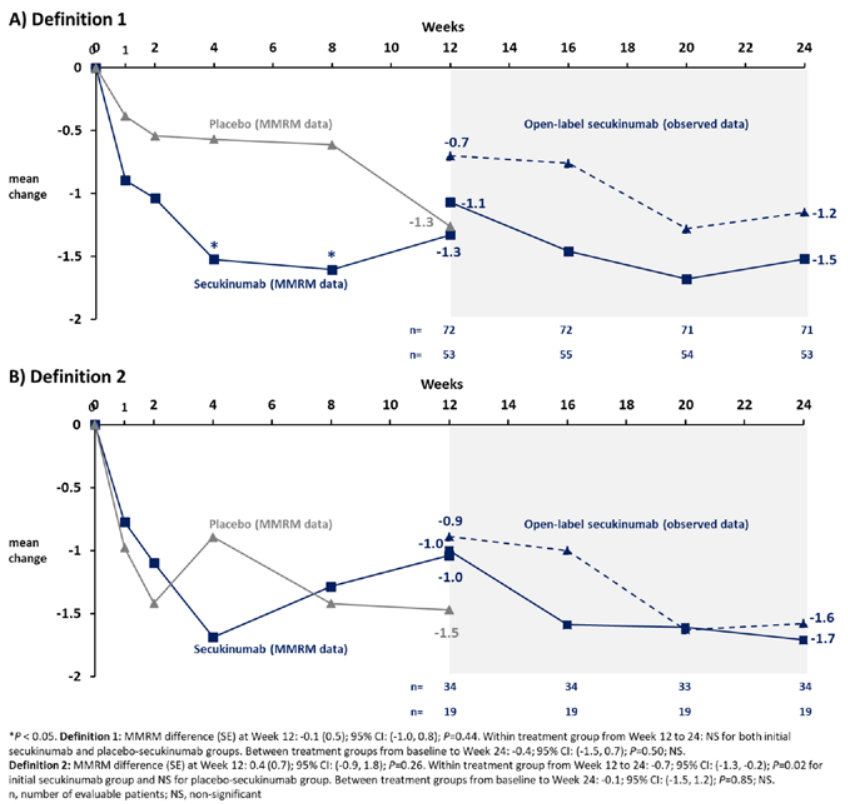

DOI: 10.1136/annrheumdis-2021-eular.1702

\section{OP0227 EFFICACY OF DEUCRAVACITINIB, AN ORAL SELECTIVE TYROSINE KINASE 2 INHIBITOR, IN MUSCULOSKELETAL MANIFESTATIONS OF ACTIVE PSORIATIC ARTHRITIS IN A PHASE 2, RANDOMIZED, DOUBLE-BLIND, PLACEBO-CONTROLLED TRIAL}

P. J. Mease ${ }^{1}$, A. Deodhar ${ }^{2}$, D. Van der Heijde ${ }^{3}$, F. Behrens ${ }^{4}$, A. Kivitz ${ }^{5}$ T. Lehman ${ }^{6}$, L. Wei ${ }^{7}$, M. Nys ${ }^{8}$, S. Banerjee ${ }^{9}$, M. Nowak ${ }^{10} .{ }^{1}$ Swedish Medical Center/Providence St. Joseph Health and University of Washington, Rheumatology Research, Seattle, United States of America; ${ }^{2}$ Oregon Health \& Science University, Division of Arthritis and Rheumatic Diseases, Portland, United States of America; ${ }^{3}$ Leiden University Medical Center, Rheumatology, Leiden, Netherlands; ${ }^{4}$ CIRI/Rheumatology and Fraunhofer Institute, Goethe University, Translational Medicine and Pharmacology ITMP, Frankfurt, United States of America; ${ }^{5}$ Altoona Center for Clinical Research, Department of Rheumatology, Duncansville, United States of America; ${ }^{6}$ Bristol Myers Squibb, Rheumatology, Princeton, United States of America; ${ }^{7}$ Bristol Myers Squibb, Medical Immunology, Princeton, United States of America; ${ }^{8}$ Bristol Myers Squibb, GBS Biostatistics, Braine-l'Alleud, Belgium; ${ }^{9}$ Bristol Myers Squibb, Rheumatology and Dermatology, Princeton, United States of America; ${ }^{10}$ Bristol Myers Squibb, Clinical R\&D, Princeton, United States of America

Background: Tyrosine kinase 2 (TYK2) is an intracellular kinase that mediates IL-23, IL-12, and IFN $\alpha / \beta$ signaling. Deucravacitinib is a novel, oral selective inhibitor of TYK2 acting via the TYK2 regulatory domain. Phase 2 results showed deucravacitinib was efficacious and well tolerated versus placebo (PBO) in patients with active psoriatic arthritis (PsA)

Objectives: This analysis further evaluated improvements in musculoskeletal disease manifestations in patients in the Phase 2 PsA trial.

Methods: The ongoing Phase 2 trial (NCT03881059) enrolled patients who had a PsA diagnosis for $\geq 6$ months, met CASPAR criteria, had active disease ( $\geq 3$ tender joints, $\geq 3$ swollen joints, C-reactive protein $[C R P] \geq 3 \mathrm{mg} / \mathrm{L}$ ), and had at least 1 active skin lesion. Patients either failed or were intolerant to at least 1 nonsteroidal anti-inflammatory drug, corticosteroid, conventional synthetic disease-modifying antirheumatic drug, and/or 1 TNF inhibitor (TNFi; $\leq 30 \%)$. Patients were randomized 1:1:1 to deucravacitinib $6 \mathrm{mg}$ QD or $12 \mathrm{mg}$ QD or PBO, and stratified by TNFi status (experienced vs naive) and body weight ( $<90 \mathrm{vs} \geq 90 \mathrm{~kg}$ ). The primary endpoint, ACR20 response at Week 16, was met and significant improvements in enthesitis vs PBO were observed. The current prespecified subgroup analysis assessed the likelihood of achieving ACR20 response at Week 16 based on study stratification factors. A post hoc analysis evaluated mean change from baseline to Week 16 in ACR components (tender joint count, swollen joint count, Physician's Global Assessment of PsA, Patients' Global Assessment of disease activity, Patients' Global Assessment of pain, high-sensitivity CRP [hCRP], and HAQ-DI score). Analyses were descriptive using data as observed.

Results: Patients treated with deucravacitinib were numerically more likely to achieve ACR20 response at Week 16 compared with PBO-treated patients regardless of TNFi experience or body weight, although some of these groups were small (Figure). Improvements for deucravacitinib $6 \mathrm{mg}$ and $12 \mathrm{mg}$ QD versus PBO were observed in all ACR components, with apparent separation occurring as early as Week 4 on, for example, HAQ-DI (mean change from baseline, -0.2 vs -0.2 vs -0.1 , respectively) and hCRP (mean change from baseline, -7.4 vs -5.2 vs 0.3 , respectively) and maintained through Week 16 (Table).

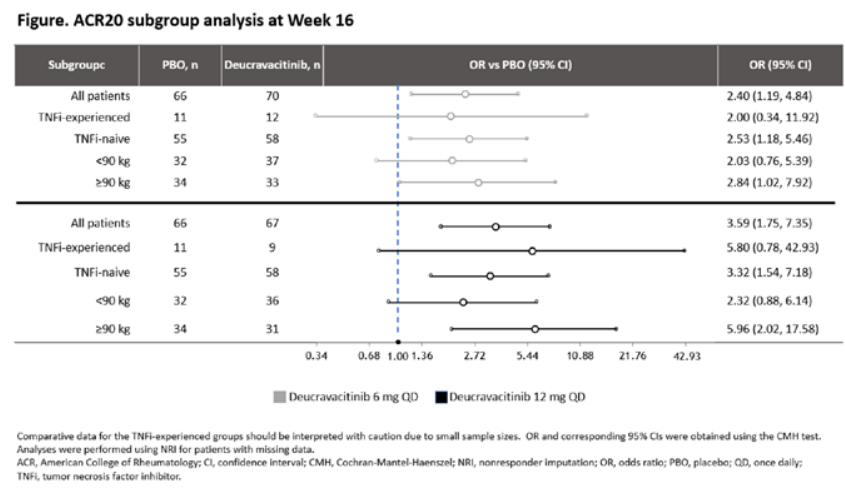

Table 1. Mean (SD) change from baseline for ACR components

\begin{tabular}{lccccccc}
\hline & TJC & SJC & PtGA & Pain & PGA & HAQ-DI & hCRP \\
\hline Baseline $^{\mathrm{a}}$ & & & & & & & \\
PBO & $16.9(9.8)$ & $10.5(7.7)$ & $66.2(15.8)$ & $64.9(18.2)$ & $63.8(14.8)$ & $1.3(0.6)$ & $20.4(39.1)$ \\
DEUC 6 & $18.1(10.3)$ & $11.9(7.0)$ & $68.2(16.8)$ & $63.6(21.7)$ & $68.2(14.7)$ & $1.3(0.6)$ & $17.6(23.6)$ \\
DEUC12 & $19.4(11.8)$ & $11.3(9.0)$ & $63.6(15.6)$ & $63.8(15.9)$ & $63.3(16.1)$ & $1.3(0.6)$ & $16.5(21.7)$ \\
Week 16 $^{\text {b }}$ & & & & & & & \\
PBO & $-4.6(9.7)$ & $-4.3(8.0)$ & $-13.4(23.5)$ & $-13.8(21.5)$ & $-19.9(21.8)$ & $-0.1(0.4)$ & $-3.3(22.6)$ \\
DEUC 6 & $-9.3(9.7)$ & $-7.7(5.8)$ & $-28.7(23.1)$ & $-25.3(26.1)$ & $-33.6(23.0)$ & $-0.4(0.5)$ & $-14.2(24.5)$ \\
DEUC 12 & $-12.2(10.2)$ & $-8.5(9.1)$ & $-27.6(25.8)$ & $-27.5(25.0)$ & $-32.2(25.0)$ & $-0.4(0.6)$ & $-10.9(22.8)$ \\
\end{tabular}

PBO, $n / N=58 / 66 ;$ DEUC $6, n / N=63 / 70 ;$ DEUC $12, n / N=59 / 67 ; n / N=$ number of patients who completed treatment/number of patients randomized; the number of patients with data avail able for individual components at each time point may vary. ${ }^{\mathrm{a}}$ Mean (SD). ${ }^{\mathrm{b}}$ Mean (SD) change from baseline.ACR, American College of Rheumatology; DEUC 6, deucravacitinib $6 \mathrm{mg}$ QD DEUC 12, deucravacitinib $12 \mathrm{mg}$ QD; HAQ-DI, Health Assessment Questionnaire-Disability Index total score; hCRP, high-sensitivity C-reactive protein; PBO, placebo; PGA, Physician's Global Assessment of psoriatic arthritis; PtGA, Patients' Global Assessment of disease activity; QD, once daily; SJC, swollen joint count; TJC, tender joint count.

Conclusion: Analyses confirmed the efficacy of deucravacitinib versus PBO across TNFi and body weight subgroups. With deucravacitinib treatment, improvements were displayed in all ACR components.

Acknowledgements: This study was sponsored by Bristol Myers Squibb. Professional medical writing assistance was provided by Peloton Advantage, LLC, an OPEN Health company, and funded by Bristol Myers Squibb.

Disclosure of Interests: Philip J Mease Consultant of: AbbVie, Amgen Boehringer Ingelheim, Bristol Myers Squibb, Eli Lilly, Galapagos, Gilead, GlaxoSmithKline, Janssen, Novartis, Pfizer, SUN Pharma, UCB, Grant/research support from: AbbVie, Amgen, Boehringer Ingelheim, Bristol Myers Squibb, Eli Lilly, Galapagos, Gilead, GlaxoSmithKline, Janssen, Novartis, Pfizer, SUN Pharma, UCB, Atul Deodhar Consultant of: AbbVie, Amgen, Boehringer 
Ingelheim, Bristol Myers Squibb, Celgene, Eli Lilly, Galapagos, GlaxoSmithKline, Janssen, Novartis, Pfizer, UCB, Grant/research support from: AbbVie, Eli Lilly, GlaxoSmithKline, Novartis, Pfizer, UCB, Désirée van der Heijde Consultant of: AbbVie, Amgen, Astellas, AstraZeneca, Bayer, Bristol Myers Squibb, Boehringer Ingelheim, Celgene, Cyxone, Daiichi, Eisai, Eli Lilly, Galapagos, Gilead, GlaxoSmithKline, Janssen, Merck, Novartis, Pfizer, Regeneron, Roche, Sanofi, Takeda, UCB Pharma. Director of Imaging Rheumatology BV, Frank Behrens Consultant of: Pfizer, AbbVie, Sanofi, Lilly, Novartis, Genzyme, Boehringer, Janssen, MSD, Celgene, Roche, Chugai, Bristol Myers Squibb, UCB Pharma, Grant/research support from: Pfizer, Janssen, Chugai, Celgene, Roche, Alan Kivitz Shareholder of: Pfizer, Sanofi, GlaxoSmithKline, Gilead Sciences, Inc., Novartis; Paid consultant: AbbVie, Boehringer Ingelheim, Flexion, Janssen, Pfizer, Sanofi, Regeneron, SUN Pharma Advanced Research, Gilead Sciences, Inc, Speakers bureau: Amgen, Horizon, Lilly, Novartis, Pfizer, Sanofi, Genzyme, Flexion, AbbVie, Thomas Lehman Shareholder of: Bristol Myers Squibb, Employee of: Bristol Myers Squibb, Lan Wei Shareholder of: Bristol Myers Squibb, Employee of: Bristol Myers Squibb, Marleen Nys Shareholder of: Bristol Myers Squibb, Employee of: Bristol Myers Squibb, Subhashis Banerjee Shareholder of: Bristol Myers Squibb, Employee of: Bristol Myers Squibb, Miroslawa Nowak Shareholder of: Bristol Myers Squibb, Employee of: Bristol Myers Squibb

DOI: 10.1136/annrheumdis-2021-eular.2653

\section{OP0228 \\ EFFICACY AND SAFETY OF RISANKIZUMAB FOR ACTIVE PSORIATIC ARTHRITIS, INCLUDING PATIENTS WITH INADEQUATE RESPONSE OR INTOLERANCE TO BIOLOGIC THERAPIES: 24-WEEK RESULTS FROM THE PHASE 3, RANDOMIZED, DOUBLE-BLIND, KEEPSAKE 2 TRIAL}

A. Ostor ${ }^{1}$, F. Van den Bosch ${ }^{2}$, K. Papp ${ }^{3}$, C. Asnal ${ }^{4}$, R. Blanco ${ }^{5}$, J. Aelion ${ }^{6}$, G. Alperovich ${ }^{7}$, Y. Zhang ${ }^{8}$, Z. Wang ${ }^{8}$, A. M. Soliman ${ }^{8}$, A. Eldred ${ }^{8}$, A. Kivitz ${ }^{9}$. ${ }^{1}$ Monash University, Cabrini Hospital, and Emertius Research, Melbourne, Australia; ${ }^{2}$ Ghent University, Department of Rheumatology, Gent, Belgium ${ }^{3}$ Probity Medical Research, K. Papp Clinical Research, Waterloo, Canada; ${ }^{4}$ Buenos Aires, DOM Centro de Reumatología, Buenos Aires, Argentina; ${ }^{5}$ Hospital Universitario Marqués de Valdecilla, IDIVAL, Santander, Spain; ${ }^{6}$ West Tennessee Research Institute, Not Applicable, Jackson, United States of America; ${ }^{7}$ AbbVie, Inc, Not Applicable, Madrid, Spain; ${ }^{8}$ AbbVie, Inc, Not Applicable, North Chicago, United States of America; ${ }^{9}$ Altoona Center for Clinical Research, Not Applicable, Duncansville, United States of America

Background: Risankizumab (RZB) is a humanized immunoglobin G1 monoclonal antibody that specifically inhibits interleukin 23 by binding its $p 19$ subunit. RZB is being investigated as a treatment for adults with psoriatic arthritis (PsA). Objectives: To compare the efficacy and safety of RZB vs placebo (PBO) for the treatment of active PsA in patients who have had inadequate response or intolerance to 1 or 2 biologic therapies (Bio-IR) or to $\geq 1$ conventional synthetic disease modifying antirheumatic drug (cSDMARD-IR).

Methods: KEEPsAKE 2 (NCT03671148) enrolled adults with active PsA ( $\geq$ 5 swollen joints [SJC] and $\geq 5$ tender joints [TJC]) who were Bio-IR or cSDMARD-IR. Patients were randomized to receive blinded subcutaneous RZB $150 \mathrm{mg}$ or PBO at weeks 0,4 , and 16 . The primary endpoint was the proportion of patients achieving $\geq 20 \%$ improvement in American College of Rheumatology score (ACR20) at week 24. Ranked secondary endpoints and other secondary endpoints are shown in the Table. Safety was assessed throughout the study. Results reported here are from the 24-week double-blind period; the open-label period with all patients receiving RZB is ongoing. Results: A total of 443 patients (RZB, $N=224 ; \mathrm{PBO}, \mathrm{N}=219$ ) were included in the analysis. Demographics and baseline disease characteristics were similar across treatment arms (mean SJC: 13.3; mean TJC: 22.6; mean duration of PsA: 8.2 years; mean body surface area involved with psoriasis $[B S A]$ in patients with BSA $\geq 3 \%: 12.1 \%)$; 206 patients $(46.5 \%)$ were Bio-IR. Significantly greater proportions of RZB-treated patients vs PBO-treated patients achieved the primary endpoint ( $51.3 \%$ vs $26.5 \%$, respectively; $P<.001)$ and all ranked secondary endpoints $(P<.001$ for all except for Functional Assessment of Chronic Illness Therapy-Fatigue [FACIT-Fatigue; $P<.009$ ]; Table). Other secondary outcomes also showed improvement for RZB- vs PBO-treated patients (Table). Serious adverse events were reported for $4.0 \%$ and $5.5 \%$ of RZB- and PBO-treated patients, respectively; serious infections were reported for $0.9 \%$ and $2.3 \%$.

Conclusion: RZB resulted in significantly greater improvements in signs and symptoms of PsA compared with PBO and was well tolerated in patients who were Bio-IR or csDMARD-IR.
Table. Efficacy Results

\begin{tabular}{|c|c|c|c|c|}
\hline & $\begin{array}{c}\text { RZB } 150 \mathrm{mg} \\
\mathrm{N}=224\end{array}$ & $\begin{array}{c}\text { PBO } \\
N=219\end{array}$ & $\begin{array}{c}\text { Difference } \\
(95 \% \mathrm{Cl})\end{array}$ & $P$ value ${ }^{\mathrm{a}}$ \\
\hline \multicolumn{5}{|l|}{ Primary endpoint } \\
\hline ACR20, \% & 51.3 & 26.5 & $24.5(15.9,33.0)$ & $<.001^{* * *}$ \\
\hline \multicolumn{5}{|l|}{ Ranked secondary endpoints } \\
\hline HAQ-DI score, change & -0.22 & -0.05 & $-0.16(-0.26,-0.07)$ & $<.001^{\star \ldots *}$ \\
\hline PASI 90,a $\%$ & 55.0 & 10.2 & $44.3(33.9,54.6)$ & $<.001^{* \ldots *}$ \\
\hline ACR20 at week $16, \%$ & 48.3 & 25.3 & $22.6(13.9,31.2)$ & $<.001^{* \times *}$ \\
\hline MDA, \% & 25.6 & 11.4 & $14.0(7.0,21.0)$ & $<.001^{\star \star * *}$ \\
\hline SF-36 PCS score, change & 5.9 & 2.0 & $3.9(2.4,5.3)$ & $<.001^{\star \star *}$ \\
\hline FACIT-Fatigue score, change & 4.9 & 2.6 & $2.2(0.6,3.9)$ & $.009^{\star \star}$ \\
\hline \multicolumn{5}{|l|}{ Other secondary endpoints } \\
\hline ACR50, \% & 26.3 & 9.3 & $16.6(9.7,23.6)$ & $<.001^{\dagger}$ \\
\hline ACR70, \% & 12.0 & 5.9 & $6.0(0.8,11.3)$ & $.024^{\dagger}$ \\
\hline Resolution of enthesitis, ${ }^{a} \%$ & 42.9 & 30.4 & $13.8(3.5,24.2)$ & $.009^{\dagger}$ \\
\hline Resolution of dactylitis, ${ }^{a} \%$ & 72.5 & 42.1 & $38.8(22.9,54.8)$ & $<.001^{\dagger}$ \\
\hline $\begin{array}{l}\text { ACR20/ACR50/ACR70, } \geq 20 / 50 / 70 \% \text { im } \\
\text { Functional Assessment of Chronic llines } \\
\text { Index; MDA, minimal disease activity, P, } \\
\text { RZB, risankizumab; SF-36 PCS, 36-Iten } \\
\text { All endpoints assessed at week } 24 \text { unles } \\
\cdots \text { : statistically significant at } 0.001 \text { level, } \\
\cdots: \text { Statistically significant at } 0.01 \text { level; } \\
\text { i: Nominal P value. }\end{array}$ & $\begin{array}{l}\text { ovement in America } \\
\text { Therapy-Fatigue; HA } \\
90, \geq 90 \% \text { reductic } \\
\text { Short Form Health S } \\
\text { otherwise noted. All } \\
\text { hultiplicity controlled. } \\
\text { Itiplicity controlled. }\end{array}$ & $\begin{array}{l}\text { in College of } \\
\text { AQ-DI, Healt } \\
\text { on in Psorias } \\
\text { survey Physic } \\
\text { "l changes ar }\end{array}$ & $\begin{array}{l}\text { Aseumatology score; FACIT-F } \\
\text { Assessment Questionnaire- } \mathrm{C} \text { Area Severity Index; PBO, } \\
\text { I Component Summary. } \\
\text { mean changes from baseline }\end{array}$ & $\begin{array}{l}\text { Eatigue, } \\
\text { Disability } \\
\text { lacebo; }\end{array}$ \\
\hline
\end{tabular}

$P B O N=158)$, or dactylitis $(R Z B N=40 ; P B O N=57)$ at baseline.

Disclosure of Interests: Andrew Ostor Speakers bureau: AÖ has received speaker or consulting fees and/or research grants from AbbVie, Bristol-Myers Squibb, Celgene, Janssen, Lilly, Merck, Novartis, Pfizer, Roche, Sanofi, and UCB., Consultant of: AÖ has received speaker or consulting fees and/or research grants from AbbVie, Bristol-Myers Squibb, Celgene, Janssen, Lilly, Merck, Novartis, Pfizer, Roche Sanofi, and UCB., Grant/research support from: AÖ has received speaker or consulting fees and/or research grants from AbbVie, Bristol-Myers Squibb, Celgene Janssen, Lilly, Merck, Novartis, Pfizer, Roche, Sanofi, and UCB., Filip van den Bosch Speakers bureau: FVdB has received speaker and/or consulting fees from Abbvie Celgene, Galapagos, Gilead, Janssen, Lilly, Novartis, Pfizer, and UCB., Consultant of: FVdB has received speaker and/or consulting fees from Abbvie, Celgene Galapagos, Gilead, Janssen, Lilly, Novartis, Pfizer, and UCB., Kim Papp Speakers bureau: KP has received honoraria or fees for serving on advisory boards, as a speaker, and as a consultant, as well as grants as principal investigator from AbbVie, Amgen, Astellas, Bausch Health (Valeant), Baxalta, Baxter, Boehringer Ingelheim, Bristol-Myers Squibb, Celgene, Coherus, Dermira, EMD Serono, Forward Pharma Galderma, Genentech, GlaxoSmithKline, Janssen, Kyowa Kirin, LEO Pharma Lilly, Medlmmune, Merck, Novartis, Pfizer, Regeneron, Roche, Sanofi Genzyme Stiefel, Sun Pharma, Takeda, and UCB., Consultant of: KP has received honoraria or fees for serving on advisory boards, as a speaker, and as a consultant, as well as grants as principal investigator from AbbVie, Amgen, Astellas, Bausch Health (Valeant), Baxalta, Baxter, Boehringer Ingelheim, Bristol-Myers Squibb, Celgene Coherus, Dermira, EMD Serono, Forward Pharma, Galderma, Genentech, GlaxoSmithKline, Janssen, Kyowa Kirin, LEO Pharma, Lilly, Medlmmune, Merck, Novartis, Pfizer, Regeneron, Roche, Sanofi Genzyme, Stiefel, Sun Pharma, Takeda, and UCB., Grant/research support from: KP has received honoraria or fees for serving on advisory boards, as a speaker, and as a consultant, as well as grants as principal investigator from AbbVie, Amgen, Astellas, Bausch Health (Valeant), Baxalta, Baxter, Boehringer Ingelheim, Bristol-Myers Squibb, Celgene, Coherus, Dermira EMD Serono, Forward Pharma, Galderma, Genentech, GlaxoSmithKline, Janssen, Kyowa Kirin, LEO Pharma, Lilly, Medlmmune, Merck, Novartis, Pfizer, Regeneron, Roche, Sanofi Genzyme, Stiefel, Sun Pharma, Takeda, and UCB., CECILIA ASNAL Speakers bureau: CA has received honoraria or fees for serving on advisory boards or as a speaker, as well as research support from AbbVie, Genentech, Janssen, Lilly, Pfizer, and Roche., Grant/research support from: CA has received honoraria or fees for serving on advisory boards or as a speaker, as well as research support from AbbVie, Genentech, Janssen, Lilly, Pfizer, and Roche., Ricardo Blanco Speakers bureau: RB has received grants or research support from AbbVie, Merck, and Roche; and has received consultation fees or honoraria for serving as a speaker for AbbVie, Bristol-Myers Squibb, Janssen, Lilly, Merck, Pfizer, and Roche., Consultant of: RB has received grants or research support from AbbVie, Merck, and Roche; and has received consultation fees or honoraria for serving as a speake for AbbVie, Bristol-Myers Squibb, Janssen, Lilly, Merck, Pfizer, and Roche., Grant/ 